\title{
Isoperimetric Type Inequalities and Hypersurface Flows
}

\author{
Pengfei Guan ${ }^{1, *}$ and Junfang $\mathrm{Li}^{2}$ \\ ${ }^{1}$ Department of Mathematics and Statistics, McGill University, Montreal, \\ Quebec H3A 0B9, Canada; \\ ${ }^{2}$ Department of Mathematics, University of Alabama at Birmingham, Birmingham, \\ Al 35294, USA.
}

Received November 11, 2019; Accepted January 10, 2020;

Published online January 12, 2021.
Dedicated to Professors Sun-Yung Alice Chang and Paul C. Yang on their 70th birthdays

\begin{abstract}
New types of hypersurface flows have been introduced recently with goals to establish isoperimetric type inequalities in geometry. These flows serve as efficient paths to achieve the optimal solutions to the problems of calculus of variations in geometric setting. The main idea is to use variational structures to develop hypersurface flows which are monotonic for the corresponding curvature integrals (including volume and surface area). These new geometric flows pose interesting but challenging PDE problems. Resolution of these problems have significant geometric implications.
\end{abstract}

AMS subject classifications: 53C23, 53C42, 35J60

Key words: Hypersurface curvature flows, geometric inequalities, quermassintegrals.

\section{Introduction}

It has been observed that the isoperimetric difference is decreasing along the curve shortening flow [9]

$$
X_{t}=-\kappa \nu,
$$

where $\kappa$ is the curvature of the boundary and $v$ the outer normal. Let $|\Omega|$ and $|\partial \Omega|$ be the area and perimeter of a bounded domain $\Omega \subset \mathbb{R}^{2}$. Along the curve shortening flow, let $\Omega(t)$ be the domain at time $t$. It follows from the Gauss-Bonnet Theorem and the Cauchy-Schwarz inequality that, the isoperimetric difference

$$
\mathcal{D}(\Omega(t))=|\partial \Omega(t)|^{2}-4 \pi|\Omega(t)|
$$

\footnotetext{
${ }^{*}$ Corresponding author. Email addresses: guan@math.mcgill.ca (P. Guan), jfli@uab.edu (J. Li)
} 
is monotonic decreasing (and strictly decreasing if $\Omega(t)$ is not a round ball). The convergence of curve shorting flow (1.1) yields the classical isoperimetric inequality in $\mathbb{R}^{2}$.

If $\Omega_{0} \subset \mathbb{N}$ is an optimal domain in a space $\mathbb{N}$ of the isoperimetric problem, then $\partial \Omega_{0}$ is a hypersurface of constant mean curvature. The isoperimetric problem can be considered as a problem of calculus of variation: for given $A$, find a domain $\Omega$ such that $\mathcal{V}(\Omega)=|\Omega|$ is of least volume among all domains in $\mathbb{N}$ with $\mathcal{A}(\Omega)=|\partial \Omega|=A$. We search for an effective path under volume constraint to achieve an optimal domain. For any variational vector field $\eta$, let $f v$ be its normal component. Then

$$
\delta_{\eta} \mathcal{V}=\int_{\partial \Omega} f d \mu_{g}
$$

where $g$ is the induced metric of the boundary $\partial \Omega$. The volume is preserved if and only if

$$
\int_{\partial \Omega} f d \mu_{g}=0 .
$$

That is, the normal component $f$ is orthogonal to the kernel of $\Delta_{g}$. This is the case if and only if

$$
f=\Delta_{g} \Phi,
$$

for some $\Phi$ on $\partial \Omega$.

One has freedom to pick any $\Phi$. We would like to search $\Phi$ such that to ensure the monotonicity of the hypersurface area. Since $\Omega$ may evolve, we look for $\Phi$ which is defined in $\mathbb{N}$ (or a region of $\mathbb{N}$ ).

Let's first consider $\mathbb{N}=\mathbb{R}^{n+1}$. For any bounded domain $\Omega \subset \mathbb{R}^{n+1}$ with smooth boundary $\partial \Omega$, let $X$ denote the position vector of the boundary surface, and let $|X|$ be the distance from the origin. In (1.2), we choose

$$
\Phi=\frac{|X|^{2}}{2} .
$$

Set $u=\langle X, v\rangle$ as the support function of $\partial \Omega$,

$$
\Delta_{g} \Phi=\Delta_{g} \frac{|X|^{2}}{2}=n-H u .
$$

This yields mean curvature type flow introduced in [15],

$$
\partial_{t} X=(n-H u) v
$$

Indeed, function $\Phi=\frac{|X|^{2}}{2}$ carries some very special geometric properties:

$$
\nabla_{g}^{2} \Phi=\nabla_{g}^{2} \frac{|X|^{2}}{2}=g-u h
$$


where $h$ is the second fundamental form of $\partial \Omega$. Denote $\sigma_{k}\left(\lambda_{1}, \cdots, \lambda_{n}\right)$ to be the $k$-th elementary symmetric function defined in $\mathbb{R}^{n}$, which can be extended to be defined in $n \times n$ symmetric matrices $h=\left(h_{i j}\right)$. Denote

$$
\sigma_{k}^{i j}(h)=\frac{\partial \sigma_{k}(h)}{\partial h_{i j}} .
$$

Set

$$
W=\sigma_{k}^{i j}(h) \nabla_{j} \Phi .
$$

Contracting $\sigma^{i j}(h)$ with $\nabla_{g}^{2} \Phi$ in (1.4),

$$
\operatorname{div} W=\nabla_{i}\left(\sigma_{k}^{i j}(h) \nabla_{j} \Phi\right)=(n-k+1) \sigma_{k-1}(h)-k u \sigma_{k}(h) .
$$

where we have used $\sigma_{k}^{i j}$ is divergent free and $h$ is Codazzi. Integrating (1.6) over $\partial \Omega$, we have the well-known Minkowski identity,

$$
(n-k+1) \int_{\partial \Omega} \sigma_{k-1}(h) d \mu_{g}=k \int_{\partial \Omega} u \sigma_{k}(h) d \mu_{g} .
$$

This identity encodes key variational structure of the curvature integrals. Along flow (1.3), the Newton-McLaurine inequality and Minkowski identity (1.7) yield the monotonicity of the area,

$$
\frac{d \mathcal{A}}{d t}=\int_{\partial \Omega} f H d \mu_{g} \leq \int_{\partial \Omega}\left(n H-\frac{2 n}{n-1} \sigma_{2} u\right) d \mu_{g}=0 .
$$

Inequality (1.8) provides a crucial monotonicity property of flow (1.3). In [15], it was proved that the hypersurface flow (1.3) exists for all time $t>0$ and

$$
\Omega(t) \rightarrow \Omega(\infty)=B^{n+1}, \quad \text { as } t \rightarrow \infty .
$$

$B^{n+1}$ is a ball with the same volume of the initial domain. As $\mathcal{A}\left(\partial B^{n+1}\right)=c_{n} \mathcal{V}^{\frac{n}{n+1}}\left(B^{n+1}\right)$, it follows the optimal isoperimetric inequality,

$$
\mathcal{A}(\partial \Omega(0)) \geq \mathcal{A}(\partial \Omega(\infty))=c_{n} \mathcal{V}^{\frac{n}{n+1}}(\Omega(\infty))=c_{n} \mathcal{V}^{\frac{n}{n+1}}(\Omega(0)) .
$$

The monotonicity formula (1.8) implies that, if equality holds, then $\Omega$ must be a ball.

Flow (1.3) resembles certain features of the standard mean curvature flow, but it is much simpler to deal with when the domains are starshaped and the existence of the flow does not require any convexity assumption. Flow (1.3) is not equivalent to rescaled version of the mean curvature flow [22].

The next flow is a special case of the flows in our earlier work [14]. Let

$$
f=\frac{1}{H} \Delta_{g} \frac{|X|^{2}}{2}=\frac{n}{H}-u
$$


Or equivalently,

$$
f=\frac{1}{H}-\frac{u}{n}
$$

In this case, instead of the volume, the first variation of the surface area has a divergence structure. Along the following inverse mean curvature type of flow

$$
\partial_{t} X=\frac{1}{n H} \Delta_{g} \frac{|X|^{2}}{2} v=\left(\frac{1}{H}-\frac{u}{n}\right) v,
$$

the surface area is invariant,

$$
\frac{d \mathcal{A}}{d t}=\frac{1}{n} \int_{\partial \Omega} \Delta_{g} \frac{|X|^{2}}{2} d \mu_{g}=0
$$

Along flow (1.9),

$$
\frac{d \mathcal{V}}{d t}=\int_{\partial \Omega}\left(\frac{1}{H}-\frac{u}{n}\right) d \mu_{g}=\int_{\partial \Omega} \frac{1}{H} d \mu_{g}-\frac{n}{n+1} \mathcal{V} \geq 0,
$$

where the last inequality follows from Heintze-Karcher inequality see $[3,20,25,26]$. Consequently, one can deduce the sharp isoperimetric inequality if the initial $\partial \Omega$ is mean convex (this condition is not needed for flow (1.3)) and if long time existence and exponential convergence of the flow can be proved, then

$$
\mathcal{V}(\Omega(0)) \leq \mathcal{V}(\Omega(\infty))=\left(\frac{\mathcal{A}(\partial \Omega(\infty))}{c_{n}}\right)^{\frac{n+1}{n}}=\left(\frac{\mathcal{A}(\partial \Omega(0))}{c_{n}}\right)^{\frac{n+1}{n}} .
$$

In contrast to flow (1.3), flow (1.9) is a normalized flow of the standard inverse mean curvature flow [11].

There are other hypersurface flows discussed in the literature related to geometric inequalities. Most of them (e.g. $[18,19,30])$ are "normalized flows" with associated to certain geometric quantity, where there are non-local terms involved. The novelty of (1.3) is that we work directly on normalized flows. One advantage of this is that the $\mathrm{C}^{0}$ estimate is immediate by the maximum principle. Another interesting feature is the monotonic properties of all the quermassintegrals along the flow. This special feature will be figured prominently in the rest discussion of this article.

\section{Mean curvature type flows in space forms and warped product spaces}

We want to generalize the argument discussed to solve the isoperimetric problem in general spaces. Let's consider space forms. Recall the Gaussian normal coordinates for the metric of a space form

$$
d s^{2}=d \rho^{2}+\phi(\rho)^{2} d z^{2}
$$


where $d z^{2}$ is the standard reduced metric of a unit sphere in $\mathbb{R}^{n+1}$. The cases of $\phi(r)=$ $\sin \rho, \rho, \sinh \rho$ yield metrics of $\mathbb{S}^{n+1}, \mathbb{R}^{n+1}$, and $\mathbb{H}^{n+1}$ respectively. There is a natural choice of function $\Phi$ which plays a similar role as $|X|^{2} / 2$ in the Euclidean space discussed in the previous section. Let

$$
\Phi=\int_{0}^{\rho} \phi(t) d t, \quad \phi^{\prime}=\frac{d \phi}{d \rho} .
$$

Then

$$
\Delta_{g} \Phi=n \phi^{\prime}-H u,
$$

where $u=\langle D \Phi, v\rangle$ is the generalized support function. $\mathbb{R}^{n+1}$ is a special case with $\Phi=$ $|X|^{2} / 2$. This leads to consider the following flow in [15],

$$
\partial_{t} X=\left(n \phi^{\prime}-H u\right) v
$$

Since $\phi \partial_{\rho}$ is a conform Killing field,

$$
\nabla_{g}^{2} \Phi=g \phi^{\prime}-u h
$$

where $h$ is the second fundamental form of $\partial \Omega$. Since the second fundamental form in space form is Codazzi, with vector field $W$ taking the same form as in (1.5),

$$
\operatorname{div} W=\nabla_{i}\left(\sigma_{k}^{i j}(h) \nabla_{j} \Phi\right)=(n-k+1) \phi^{\prime} \sigma_{k-1}(h)-k u \sigma_{k}(h) .
$$

This immediately deduces the following Minkowski identity

$$
(n-k+1) \int_{\partial \Omega} \phi^{\prime} \sigma_{k-1}(h) d \mu_{g}=k \int_{\partial \Omega} u \sigma_{k}(h) d \mu_{g} .
$$

As in the case of $\mathbb{R}^{n+1}$, one obtains the same monotonicity formulas for the volume and hypersurface area

$$
\begin{aligned}
& \mathcal{V}(\Omega(0))=\mathcal{V}(\Omega(t))=\mathcal{V}(\Omega(\infty)), \\
& \mathcal{A}(\partial \Omega(0)) \geq \mathcal{A}(\partial \Omega(t)) \geq \mathcal{A}(\partial \Omega(\infty))
\end{aligned}
$$

Since powers of volume of balls and powers of area of the boundary spheres do not always have simple algebraic relation in space forms except in $\mathbb{R}^{n+1}$, instead of having a simple isoperimetric inequality as in $\mathbb{R}^{n+1}$, we conclude an isoperimetric comparison inequality with geodesic balls by establishing long time existence and exponential convergence [15]. If $\Omega$ has the same volume of a geodesic ball $B$,

$$
\mathcal{V}(\Omega)=\mathcal{V}(B),
$$

then the surface area of $\partial \Omega$ is not less than the area of the sphere

$$
\mathcal{A}(\partial \Omega) \geq \mathcal{A}(\partial B),
$$


with equality holds if and only if $\Omega$ is a geodesic ball.

The hypersurface flow (2.2) can be extended further to more general warped product spaces. Let $\left(\mathbf{N}^{n+1}, \bar{g}\right)$ be a Riemannian manifold with the warped product structure,

$$
\bar{g}=d \rho^{2}+\phi(\rho)^{2} \tilde{g}, \text { on }\left[r_{0}, \bar{r}\right] \times B,
$$

where $\tilde{g}$ is the Riemannian metric of the base manifold $B$ which may not necessarily be spheres. The following flow was considered in [17],

$$
\partial_{t} X=\left(n \phi^{\prime}-H u\right) v=\Delta \Phi v,
$$

where $u=\langle D \Phi, v\rangle$ and $\Phi(r)$ and $\phi^{\prime}$ are defined the same as above. Flow (2.7) preserves the volume, we need monotonicity of the area. As $\sigma_{2}^{i j}=\frac{\partial \sigma_{2}}{\partial h_{i j}}=H g^{i j}-h^{i j}$, the trace of its covariant derivative is

$$
\sigma_{2}^{i j}(h)_{j}=-\bar{R}_{i v},
$$

where $v$ is the unit outward normal of the hypersurface. The Minkowski identity (2.5) will have extra terms involving Ricci tensor of the ambient space, since $\bar{R}_{i v} \neq 0$ in general.

The Ricci curvature tensor of $\left(\mathbf{N}^{n+1}, \bar{g}\right)$ is given by

$$
\left.\bar{R} i c=-n \frac{\phi^{\prime \prime}}{\phi} d \rho^{2}-\left[(n-1) \phi^{2}\right)+\phi \phi^{\prime \prime}\right] \tilde{g}+\tilde{R} i c .
$$

For general warped product space, $X=\phi(\rho) \partial_{\rho}$ is a conformal Killing vector field, identity (2.3) still holds. We have

$$
\partial_{i}\left(\sigma_{2}^{i j}(h) \Phi_{j}\right)=(n-1) \phi^{\prime} \sigma_{1}(h)-2 u \sigma_{k}(h)-\bar{R}_{i v} \Phi_{i} .
$$

The Minkowski identity takes the following form for compact hypersurface $M \subset \mathbf{N}^{n+1}$,

$$
(n-1) \int_{M} \phi^{\prime} \sigma_{1} d \mu=2 \int_{M} u \sigma_{2} d \mu+\int_{M} \bar{R}_{i v} \Phi_{i} .
$$

If $M(t)$ is a smooth one-parameter family of closed graphical hypersurface in $\mathbf{N}^{n+1}$ which solves the parabolic equations (2.7), then

$$
\begin{aligned}
A^{\prime}(t) & =\int\left(n \phi^{\prime}-H u\right) H d \mu_{g} \\
& =\int\left(n \phi^{\prime} H-\frac{2 n}{n-1} \sigma_{2} u\right) d \mu_{g}+\int\left(\frac{2 n}{n-1} \sigma_{2}-H^{2}\right) u d \mu_{g} \\
& =\int \frac{n}{n-1} \bar{R}_{i v} \nabla_{i} \Phi d \mu_{g}+\int\left(\frac{2 n}{n-1} \sigma_{2}-H^{2}\right) u d \mu_{g} .
\end{aligned}
$$

If $K-\phi^{\prime 2}+\phi \phi^{\prime \prime} \geq 0$ and $\tilde{R i c} \geq(n-1) K \tilde{g}$, it follows from (2.9) that

$$
\bar{R}_{i v} \nabla_{i} \Phi \leq 0 .
$$


Thus, the crucial monotonicity is held,

$$
\frac{d \mathcal{A}(\partial \Omega(t))}{d t} \leq 0
$$

A solution to the isoperimetric problem for warped product spaces can be obtained as follows. Let $S(r)$ be a level set of $r$ and $B(r)$ be the bounded domain enclosed by $S(r)$ and $S\left(r_{0}\right)$. The volume of $B(r)$ and surface area of $S(r)$, both positive functions of $r$, are denoted as $\mathcal{V}(r)$ and $\mathcal{A}(r)$, respectively. Note that $\mathcal{V}=\mathcal{V}(r)$ is strictly increasing function of $r$. Consider the single variable function $\xi(x)$ that satisfies

$$
\mathcal{A}(r)=\xi(\mathcal{V}(r))
$$

for any $r \in\left[r_{0}, \bar{r}\right]$. The function $\xi(x)$ is well-defined.

Theorem 2.1 ([17]). Let $\Omega \subset \mathbf{N}^{n+1}$ be a domain bounded by a smooth graphical hypersurface $M$ and $S\left(r_{0}\right)$ with $n \geq 2$. Suppose $\partial \Omega$ is inside the region $\left[r_{0}, \bar{r}\right] \times B$ where is is the base manifold. Suppose

$$
\begin{aligned}
& \tilde{R i c} \geq(n-1) K \tilde{g}, \\
& 0 \leq\left(\phi^{\prime}\right)^{2}-\phi^{\prime \prime} \phi \leq K \text { on }\left[r_{0}, \bar{r}\right],
\end{aligned}
$$

and assume $\phi(r)$ and $\tilde{g}$ satisfy the conditions (2.14), then flow (2.7) exists all time and convergent exponentially to a slice $r=r_{1}$ for some $r_{1}$, and

$$
\operatorname{Area}(M) \geq \xi(\operatorname{Vol}(\Omega)),
$$

where $\operatorname{Area}(M)$ is the area of $M$ and $\operatorname{Vol}(\Omega)$ is the volume of $\Omega$, and function $\xi$ is defined in (2.13). If, in addition to (2.14), either $\left(\phi^{\prime}\right)^{2}-\phi^{\prime \prime} \phi<K$ or Ric $>(n-1) K \tilde{g}$ on $\left[r_{0}, \bar{r}\right]$ then " $="$ is attained in (2.15) if and only if $M$ is a level set of $r$.

The condition $\left(\phi_{r}\right)^{2}-\phi_{r r} \phi \leq K$ is necessary since this is equivalent to the condition of stability of slice $\{r=c\}$ as a hypersurface of constant mean curvature. The condition $0 \leq\left(\phi_{r}\right)^{2}-\phi_{r r} \phi$ is imposed in [17] for the gradient estimates of PDE of the radial function. Note that for flows (1.3), (2.2) and (2.7), $\partial \Omega$ is not assumed to any convex assumptions.

\section{Fully nonlinear flows and quermassintegrals in $\mathbb{R}^{n+1}$}

We consider hypersurface flows related to the quermassintegrals. In convex geometry, there is the notion of quermassintegrals. If $\Omega \subset \mathbb{R}^{n+1}$ is a $C^{2}$ domain, the quermassintegrals can be expressed in terms of boundary curvature integrals:

$$
\begin{aligned}
& \mathcal{A}_{-1}(\Omega)=\mathcal{V}(\Omega), \quad \mathcal{A}_{0}(\Omega)=\mathcal{A}(\Omega), \\
& \mathcal{A}_{k}(\Omega)=\int_{\partial \Omega} \sigma_{k}(\kappa) d \mu_{g}, \quad k=1, \cdots, n-1,
\end{aligned}
$$


where $\kappa=\left(\kappa_{1}, \cdots, \kappa_{n}\right)$ the principal curvatures of $\partial \Omega$. The classical Alexandrov-Fenchel inequality states that, there is dimensional constants $C(n, k)$ such that for all compact convex domain $\Omega \subset \mathbb{R}^{n+1}$,

$$
\mathcal{A}_{k-1}(\Omega) \leq C(n, k)\left(\mathcal{A}_{k}(\Omega)\right)^{\frac{n+1-k}{n-k}}, \quad \forall k=0, \cdots, n-1,
$$

with equality holds if and only if $\Omega$ is a ball.

We want to design hypersurface flow as path to the optimal solution to inequality (3.1). Set

$$
c_{n, k}=\frac{\sigma_{k+1}}{\sigma_{k}}(I) .
$$

The $l$-th quermassintegral enjoys the following variational property, for any variational vector field $\eta=f v$,

$$
\delta_{\eta} \mathcal{A}_{l}(\Omega)=(l+1) \int_{\partial \Omega} f \sigma_{l+1}(\kappa) d \mu
$$

Suppose

$$
f=\frac{\tilde{f}}{\sigma_{k+1}(\kappa)}
$$

by (3.3),

$$
\delta_{\eta} \mathcal{A}_{k}(\Omega)=(l+1) \int_{\partial \Omega} \tilde{f} d \mu .
$$

If we want to have $\mathcal{A}_{k}$ preserved, we should look for speed function

$$
f=\frac{\operatorname{div} W}{\sigma_{k+1}}
$$

for some vector field $W$. In this case, a natural choice of $W$ is given in (1.5), that is

$$
W=\sigma_{k}^{i j} \nabla_{j} \Phi
$$

This leads us to consider the following inverse mean curvature type flow

$$
\partial_{t} X=\left(\frac{\sigma_{k}}{\sigma_{k+1}}(\kappa)-\frac{u}{c_{n, k}}\right) v=\frac{\operatorname{div} W}{\sigma_{k+1}} v
$$

so that $\mathcal{A}_{k}$ is preserved. By the Minkowski identity (2.5) and the Newton-McLaurine inequality,

$$
\frac{d \mathcal{A}_{k+1}(\Omega)}{d t}=(k+2) \int_{\partial \Omega}\left(\frac{\sigma_{k}}{\sigma_{k+1}}(\kappa)-\frac{u}{c_{n, k}}\right) \sigma_{k+2}(\kappa) d \mu \leq 0 .
$$


The following monotonicity properties hold,

$$
\begin{aligned}
& \mathcal{V}(\Omega(0)) \leq \mathcal{V}(\Omega(t)) \leq \mathcal{V}(\Omega(\infty)) \\
& \mathcal{A}(\partial \Omega(0)) \leq \mathcal{A}(\partial \Omega(t)) \leq \mathcal{A}(\partial \Omega(\infty)) \\
& \ldots \\
& \mathcal{A}_{k}(\partial \Omega(0))=\mathcal{A}_{k}(\partial \Omega(t))=\mathcal{A}_{k}(\partial \Omega(\infty)) \\
& \mathcal{A}_{k+1}(\partial \Omega(0)) \geq \mathcal{A}_{k+1}(\partial \Omega(t)) \geq \mathcal{A}_{k+1}(\partial \Omega(\infty)),
\end{aligned}
$$

The sharp Alexandrov-Fenchel inequalitites for starshaped $(k+1)$-convex domains was established in [14] along this way. Recall that, $\Omega$ is $l$-convex if the principal curvature of $\partial \Omega, \kappa \in \Gamma_{l}$, , where $\Gamma_{l}$ is the Garding cone

$$
\Gamma_{l}=\left\{\Lambda \in \mathbb{R}^{n} \mid \sigma_{1}(\Lambda)>0, \cdots, \sigma_{l}(\Lambda)>0\right\}
$$

Flow (3.4) is equivalent to the inverse curvature flow $\partial_{t} X=\frac{\sigma_{k}}{\sigma_{k+1}} v$ introduced by Gerhardt [11] by rescaling, see also [13].

One may also design a flow such that $\mathcal{A}_{k-1}$ is preserved as follow [16]

$$
\partial_{t} X=\left(c_{n, k}-\frac{\sigma_{k+1}}{\sigma_{k}} u\right) v=\frac{\operatorname{div} W}{\sigma_{k}} v,
$$

where the vector field $W$ is again as in (1.5). When $k=0,(3.5)$ reduces to the mean curvature type flow (1.3).

If flow (3.5) exists for all $t \geq 0$ and converges to a sphere with the same $\mathcal{A}_{k-1}$ of the initial domain, we have the following monotonicity properties

$$
\begin{aligned}
& \mathcal{V}(\Omega(0)) \leq \mathcal{V}(\Omega(t)) \leq \mathcal{V}(\Omega(\infty)) \\
& \mathcal{A}(\partial \Omega(0)) \leq \mathcal{A}(\partial \Omega(t)) \leq \mathcal{A}(\partial \Omega(\infty)) \\
& \quad \cdots \\
& \mathcal{A}_{k-1}(\partial \Omega(0))=\mathcal{A}_{k-1}(\partial \Omega(t))=\mathcal{A}_{k-1}(\partial \Omega(\infty)) \\
& \mathcal{A}_{k}(\partial \Omega(0)) \geq \mathcal{A}_{k}(\partial \Omega(t)) \geq \mathcal{A}_{k}(\partial \Omega(\infty))
\end{aligned}
$$

This would immediately imply the sharp quermassintegral inequalities in convex geometry. In question is the longtime existence and convergence of flow (3.5). The major PDE problem for flow (3.5) is the curvature estimate (or $C^{2}$ estimates). To overcome this difficulty, we transform flow (3.5) to a parabolic PDE on $\mathbb{S}^{n}$ in [16]. Let $\Omega \subset \mathbb{R}^{n+1}$ be a bounded strictly convex domain with smooth boundary. Let $W_{j}^{i}$ be its Weingarten curvature tensor. It is well-known that the boundary hypersurface can be parametrized by the support function of the inverse of its Gauss map if the domain is strictly convex. Namely, the 
support function $u=u(v)$, where $v \in \mathbb{S}^{n}$ are the outward normal vector at points on the boundary. With this parametrization, the inverse of the Weingarten curvature has a simple form

$$
\left(W^{-1}\right)_{j}^{i}=e^{i k} u_{k j}+u \delta_{j}^{i}=: A_{j}^{i},
$$

where $e_{i j}$ is the metric tensor of the standard unit sphere $\mathbb{S}^{n}$. The induced metric of the hypersurface satisfies $g_{i j}:=e^{k l} A_{i k} A_{j l}$.

One can show that the following parabolic PDE of the support function $u$ is equivalent to flow (3.5) for convex domains,

$$
\partial_{t} u=c_{n, k}-\frac{u}{G}
$$

where $G=\frac{\sigma_{n-k-1}(A)}{\sigma_{n-k}(A)}$ and $c_{n, k}=\frac{1}{G(I)}$ with $I=(1, \cdots, 1)$. For Eq. (3.6), admissible solutions are for those $u$ with $A \in \Gamma_{n-k}$. In this section, we prove exponential convergence of flow (3.6).

We will assume normal coordinates of $\mathbb{S}^{n}$ and do not distinguish upper or lower indexes of the tensors. Thus the metric of $\mathbb{S}^{n}$ can be denoted as $\delta_{i j}$. Moreover, we will use Einstein's convention, i.e., repeated indexes in tensor calculations implies summation over the index. For convenience, we will use the following linearized operator,

$$
\mathcal{L}:=\partial_{t}-\frac{u}{G^{2}} \nabla_{i} \nabla_{j}
$$

$C^{0}$ estimate can be immediately obtained by maximum principle,

$$
\min _{\mathbb{S}^{n}} u(0, \cdot) \leq u(t, \cdot) \leq \max _{\mathbb{S}^{n}} u(0, \cdot),
$$

for any $t \in[0, T]$.

Let $\lambda$ be a vector and $G=\frac{\sigma_{n-k}(\lambda)}{\sigma_{n-k-1}(\lambda)}$. If $\lambda \in \Gamma_{n-k}$, then

$$
G(I) \leq G^{i i} \leq k+1, \quad \text { where } I=(1, \cdots, 1) .
$$

Let us first deal with the uniform lower bound of $G(t, \cdot)$.

Lemma 3.1. Suppose (3.6) has a solution $u(t, \cdot)$ on $[0, T] \times \mathbb{S}^{n}$, we have

$$
\min _{[0, T] \times \mathbb{S}^{n}} G(t, \cdot) \geq \min _{\mathbb{S}^{n}} G(0, \cdot)>0 .
$$

Proof. Recall $u_{t}=c_{n, k}-\frac{u}{G}$,

$$
\begin{aligned}
\partial_{t} G & =G^{i j}\left(A_{i j, t}\right)=G^{i j}\left(u_{t, i j}+u_{t} \delta_{i j}\right) \\
& =\frac{u}{G^{2}} G^{i j} G_{i j}+G^{i j}\left(\frac{u_{j} G_{i}}{G^{2}}+\frac{u_{i} G_{j}}{G^{2}}-\frac{2 u G_{i} G_{j}}{G^{3}}\right)-\frac{G^{i j}}{G} u_{i j}+G^{i j}\left(c_{n, k}-\frac{u}{G}\right) \delta_{i j},
\end{aligned}
$$


where $G^{i j}:=\frac{\partial G}{\partial A_{i j}}$ and $G_{i j}:=\nabla_{i} \nabla_{j} G$ with respect to the standard metric of $\mathbb{S}^{n}$. We can combine the last two terms in (3.9),

$$
-\frac{G^{i j}}{G} u_{i j}+G^{i j}\left(c_{n, k}-\frac{u}{G}\right) \delta_{i j}=-\frac{1}{G} G^{i j} A_{i j}+c_{n, k} G^{i i}=c_{n, k} G^{i i}-1 \geq 0 .
$$

The last inequality follows from (3.8). Putting (3.10) into (3.9),

$$
\mathcal{L}(G) \geq G^{i j}\left(\frac{u_{j} G_{i}}{G^{2}}+\frac{u_{i} G_{j}}{G^{2}}-\frac{2 u G_{i} G_{j}}{G^{3}}\right)
$$

where $\mathcal{L}$ is defined in (3.7). By applying the standard maximum principle to (3.11) on $G$, we finish the proof.

The proof for the uniform upper bound for $G$ is similar as the proof of Lemma 3.5 in [16].

Lemma 3.2. Suppose (3.6) has a solution $u(t, \cdot)$ on $[0, T] \times \mathbb{S}^{n}$, we have

$$
\max _{[0, T] \times \mathbb{S}^{n}} G(t, \cdot) \leq C,
$$

where $C$ is a constant only depends on the initial hypersurface.

Proof. We derive the evolution of $\frac{u}{G}$.

$$
\begin{aligned}
\partial_{t} \frac{u}{G} & =\frac{u_{t}}{G}-\frac{u}{G^{2}} G^{i j}\left(u_{t, i j}+u_{t} \delta_{i j}\right)=\frac{u}{G^{2}} G^{i j}\left(\frac{u}{G}\right)_{i j}+\frac{u_{t}}{G}\left(1-\frac{u}{G} G^{i i}\right) \\
& =\frac{u}{G^{2}} G^{i j}\left(\frac{u}{G}\right)_{i j}+\left(c_{n, k}-\frac{u}{G}\right) \frac{1}{G}\left(1-\frac{u}{G} G^{i i}\right) .
\end{aligned}
$$

By our $C^{0}$ estimate, $u$ is uniformly bounded from above and below by positive constants. Assume the minimum of $\frac{u}{G}>0$ is attained at $P_{0}$. Then at $P_{0}$, using critical points condition, we have

$$
0 \geq \mathcal{L}\left(\frac{u}{G}\right)=\left(c_{n, k}-\frac{u}{G}\right) \frac{1}{G}\left(1-\frac{u}{G} G^{i i}\right)
$$

Without loss of generality, we assume $\frac{u}{G}<c_{n, k}$, otherwise $\frac{u}{G} \geq c_{n, k}$ is bounded from below. Thus at $P_{0}$, from (3.13), we obtain $1-\frac{u}{G} G^{i i} \leq 0$. Namely,

$$
\frac{u}{G} \geq \frac{1}{G^{i i}} \geq \frac{1}{k+1}
$$

where the last inequality follows from Lemma 3.8. This yields $\frac{u}{G} \geq c>0$. By the uniformly boundedness of $u$, we finish the proof. 
The next lemma provides the $C^{1}$ and $C^{2}$ estimates for admissible solutions of flow (3.6).

Lemma 3.3. Suppose (3.6) has a solution $u(t, \cdot)$ on $[0, T] \times \mathbb{S}^{n}$, we have

$$
\max _{[0, T] \times \mathbb{S}^{n}}|\nabla u|^{2} \leq C, \quad \max _{[0, T] \times \mathbb{S}^{n}}|\nabla \nabla u|^{2} \leq C,
$$

where $C$ is a constant only depends on the initial hypersurface.

Proof. We first derive the evolution $|\nabla u|^{2}$.

$$
\begin{aligned}
\left(|\nabla u|^{2}\right)_{t} & =2 u_{l} u_{t l}=-2 u_{l}\left(\frac{u}{G}\right)_{l}=-2 \frac{|\nabla u|^{2}}{G}+2 \frac{u}{G^{2}} u_{l} G^{i j} A_{i j, l} \\
& =-2 \frac{|\nabla u|^{2}}{G}+2 \frac{u}{G^{2}} u_{l} G^{i j} A_{i l, j},
\end{aligned}
$$

where we used $A_{i j}$ is Codazzi in the last step. Thus

$$
\begin{aligned}
\left(|\nabla u|^{2}\right)_{t} & =-2 \frac{|\nabla u|^{2}}{G}+2 \frac{u}{G^{2}} u_{l} G^{i j}\left(u_{i l, j}+u_{j} \delta_{i l}\right) \\
& =-2 \frac{|\nabla u|^{2}}{G}+\frac{u}{G^{2}} G^{i j}\left(|\nabla u|^{2}\right)_{i j}-2 \frac{u}{G^{2}} G^{i j} u_{i l} u_{j l}+2 \frac{u}{G^{2}} G^{i j} u_{i} u_{j}
\end{aligned}
$$

Equivalently, we have

$$
\mathcal{L}\left(|\nabla u|^{2}\right)=-2 \frac{|\nabla u|^{2}}{G}-2 \frac{u}{G^{2}} G^{i j} u_{i l} u_{j l}+2 \frac{u}{G^{2}} G^{i j} u_{i} u_{j} .
$$

On the other hand, we have

$$
\begin{aligned}
\mathcal{L}\left(u^{2}\right) & =2 u\left(c_{n, k}-\frac{u}{G}\right)-\frac{u}{G^{2}} G^{i j}\left(u^{2}\right)_{i j} \\
& =2 u\left(c_{n, k}-\frac{u}{G}\right)-\frac{2 u^{2}}{G^{2}} G^{i j} u_{i j}-\frac{2 u}{G^{2}} G^{i j} u_{i} u_{j} \\
& =2 u\left(c_{n, k}-\frac{u}{G}\right)-2 \frac{u^{2}}{G}+2 \frac{u^{3}}{G^{2}} G^{i i}-\frac{2 u}{G^{2}} G^{i j} u_{i} u_{j},
\end{aligned}
$$

where we have used the following contraction,

$$
G^{i j} u_{i j}=G^{i j} A_{i j}-u G^{i i}=G-u G^{i i} .
$$

Adding (3.17) and (3.18), we obtaino

$$
\begin{aligned}
\mathcal{L}\left(|\nabla u|^{2}+u^{2}\right) & =-2 \frac{|\nabla u|^{2}}{G}-2 \frac{u}{G^{2}} G^{i j} u_{i l} u_{j l}+2 c_{n, k} u-4 \frac{u^{2}}{G}+2 \frac{u^{3}}{G^{2}} G^{i i} \\
& \leq-2 \frac{|\nabla u|^{2}}{G}+2 c_{n, k} u-4 \frac{u^{2}}{G}+2(k+1) \frac{u^{3}}{G^{2}} .
\end{aligned}
$$


where we have used $G^{i i} \leq k+1$. Since $u, G$ are uniformly bounded above and below and $G$. By standard maximum principle, we obtain the gradient estimate.

We now consider $C^{2}$ estimate. The evolution equation of $A_{i j}$ is (c.f. Proposition 3.4 in [16]),

$$
\begin{aligned}
\mathcal{L}\left(A_{i j}\right)= & \frac{u}{G^{2}} \ddot{G}\left(\nabla_{i} A, \nabla_{j} A\right)+\frac{2}{G^{2}} u_{i} G_{j}-\frac{2 u}{G^{3}} G_{i} G_{j}-\frac{1}{G}\left(\frac{u}{G} G^{k k}+1\right) A_{i j}+\left(c_{n, k}+\frac{u}{G}\right) \delta_{i j} \\
= & \frac{u}{G^{2}} \ddot{G}\left(\nabla_{i} A, \nabla_{j} A\right)-\frac{2 u}{G^{3}}\left(G_{i}-\frac{G}{2 u} u_{i}\right)\left(G_{j}-\frac{G}{2 u} u_{j}\right) \\
& -\frac{u}{G^{2}}\left(G^{k k} A_{i j}-G \delta_{i j}\right)-\frac{1}{G}\left(A_{i j}-c_{n, k} G \delta_{i j}\right)+\frac{1}{2 u G} u_{i} u_{j} \\
\leq & -\frac{u}{G^{2}}\left(G^{k k} A_{i j}-G \delta_{i j}\right)-\frac{1}{G}\left(A_{i j}-c_{n, k} G \delta_{i j}\right)+\frac{1}{2 u G} u_{i} u_{j},
\end{aligned}
$$

where we have used the concavity of $\ddot{G}$ and also removed the negative quadratic term. Suppose $\lambda_{\max }$ is the maximal eigenvalue of $A_{i j}$ for all points and $0 \leq t \leq T$. If it is attained at $t_{0} \in(0, T]$, we may assume $\lambda_{\max }=A_{11}\left(x_{0}, t_{0}\right)$, for $t_{0}>0, x_{0} \in S^{n}$. At $P_{0}$, we have

$$
0 \leq \mathcal{L}\left(A_{11}\right) \leq-\frac{u}{G^{2}}\left(G^{k k} A_{11}-G\right)-\frac{1}{G}\left(A_{11}-c_{n, k} G\right)+\frac{1}{2 u G} u_{1} u_{1} .
$$

Since

$$
G^{k k} A_{11}-G=\sum_{k k} G^{k k}\left(A_{11}-A_{k k}\right) \geq 0,
$$

we can eliminate the first term on the right side of (3.22) and obtain

$$
0 \leq-\frac{1}{G}\left(A_{11}-c_{n, k} G\right)+\frac{1}{2 u G} u_{1} u_{1} .
$$

Equivalently, we obtain

$$
A_{11} \leq c_{n, k} G+\frac{|\nabla u|^{2}}{2 u} .
$$

Since $|\nabla u|^{2}$ and $G$ are bounded from above, $u$ is bounded from below, we proved $A_{i j}$ is uniformly bounded from above. This immediately implies the desired $C^{2}$ bounds.

Without appealing to the corresponding hypersurface flow (3.5) like in [16], we will provide direct proofs for the convergence property of the flow at infinity. With $C^{2}$ estimates in hand, the Krylov Theorem yields $C^{2, \alpha}$ estimates and the higher regularity estimates follows from standard theory for parabolic equations. Therefore, flow (3.6) exists all time and one may argue that solution is sequentially convergent to solitons of the form

$$
c_{n, k}=\frac{u}{G} .
$$


Since by the Newton McLaurine inequality,

$$
0 \geq \frac{2}{c_{n, k}} H-2 n G=\frac{2}{c_{n, k}} \Delta u .
$$

This yields that $u$ is a super-harmonic function on $\mathbb{S}^{n}$. So $u$ is a constant. This implies the convergence of flow (3.6).

We will use a similar argument as in Proposition 5.5 of [15] to show that when $t$ is large enough, different eigenvalues of $A_{i j}$ at the same point are comparable uniformly for arbitrary small $\epsilon$.

Lemma 3.4. Let $u=u(t, \cdot)$ solves the initial value problem (3.6). Then for any $\epsilon>0$, there exists $T_{0}>0$, such that for any $t>T_{0}$,

$$
\max _{i<j, z \in \mathbb{S}^{n}}\left|\lambda_{i}(t, z)-\lambda_{j}(t, z)\right|<\epsilon
$$

where $\lambda(t, z)$ are the eigenvalues of $A_{i j}$ at $(t, z)$.

Proof. We have the evolution of the following integral on $\mathbb{S}^{n}$. For simplicity, we denote $\mathcal{I}:=\int_{\mathbb{S}^{n}} \sigma_{n-k}\left(A_{i j}\right) d z^{n}$, then

$$
\begin{aligned}
\frac{d}{d t} \mathcal{I}(t)= & (k+1) \int_{\mathbb{S}^{n}}\left(c_{n, k}-\frac{u \sigma_{n-k-1}}{\sigma_{n-k}}\right) \sigma_{n-k-1} d z^{n} \\
\leq & (k+1) \int_{\mathbb{S}^{n}}\left(c_{n, k} \sigma_{n-k-1}-\frac{u \sigma_{n-k-1}^{2}}{\sigma_{n-k}}\right) d z^{n} \\
\leq & (k+1) \int_{\mathbb{S}^{n}}\left[c_{n, k} \sigma_{n-k-1}-\frac{(n-k)(k+2)}{(n-k-1)(k+1)} u \sigma_{n-k-2}\right] d z^{n} \\
& +(k+1) \int_{\mathbb{S}^{n}} u\left[\frac{(n-k)(k+2)}{(n-k-1)(k+1)} \sigma_{n-k-2}-\frac{\sigma_{n-k-1}^{2}}{\sigma_{n-k}}\right] d z^{n} .
\end{aligned}
$$

The first integral in the last inequality of (3.26) vanishes due to the Minkowski identity on $S^{n}$. By the Newton-McLaurine inequality,

$$
\left[\frac{(n-k)(k+2)}{(n-k-1)(k+1)} \sigma_{n-k-2}-\frac{\sigma_{n-k-1}^{2}}{\sigma_{n-k}}\right] \leq 0
$$

pointwisely. The " $="$ is attained if and only if $\lambda_{1}=\cdots=\lambda_{n}$. We continue to argue as in [15]. We start with

$$
\mathcal{I}(t)-\mathcal{I}(0)=\int_{0}^{t} A^{\prime}(s) d s
$$


From (3.26) and (3.27), we have $\mathcal{I}^{\prime}(s) \leq 0$. By our regularity estimates, we have $\mathcal{I}(t)$ is uniformly bounded from above and below. Thus,

$$
\int_{0}^{\infty} A^{\prime}(t) d t<\infty
$$

Since we have established uniform a priori estimates for all the derivatives of $u$ for any order and also $0<C_{1} \leq u \leq C_{2}$ uniformly, $\mathcal{I}^{\prime}(t)$ is uniformly continuous with respect to $t$. On the other hand, $d \mu_{g}$ is also uniformly bounded from up and below. This proves that for any $\epsilon>0$, there exists a large enough $T_{0}>0$, such that for any $t>T_{0}$, the NewtonMacLaurin difference can be arbitrarily small. Namely,

$$
0 \leq \frac{\sigma_{n-k-1}^{2}}{\sigma_{n-k}}-\frac{(n-k)(k+2)}{(n-k-1)(k+1)} \sigma_{n-k-2} \leq \epsilon .
$$

This completes the proof of the lemma.

Lemma 3.4 yields that $A_{i j}$ is positive definite with eigenvalues bounded from below and above when $t$ large. With uniform convexity, when $t$ is large,

$$
\sum_{i=1}^{n} G-G^{11} A_{11} \geq C_{1}>0
$$

At maximum point $\left(t, x_{0}\right)$ of $|\nabla u(t, x)|$ with $\nabla u=\left(u_{1}, 0, \cdots, 0\right)$, since $u_{1 j}=0$ so that $A_{11}=u$. We may assume $G^{i j}$ diagonal, it follows from (3.17),

$$
\begin{aligned}
\mathcal{L}\left(|\nabla u|^{2}\right) & =-2\left(-\frac{|\nabla u|^{2}}{G}-\frac{u}{G^{2}} G^{i j} u_{i l} u_{j l}+\frac{u}{G^{2}} G^{i j} u_{i} u_{j}\right) \\
& \leq-2|\nabla u|^{2}\left(\frac{1}{G}-\frac{G^{11} A_{11}}{G^{2}}\right) \leq-2 C_{1} \frac{|\nabla u|^{2}}{G} .
\end{aligned}
$$

That is, $|\nabla u|^{2}$ is convergent to 0 exponentially. One may also go back to the corresponding hypersurface flow (3.5) to deduce the exponential convergence. $A_{i j}$ is uniform definite when $t$ large, thus flow (3.5) is uniformly parabolic. Then one may infer Proposition 3.1 in [16] to get the exponential convergence.

We do not know if the convexity of $A_{i j}$ is preserved along flow (3.6), this would imply the curvature estimates and longtime existence for flow (3.5).

\section{Fully nonlinear flows in $\mathbb{H}^{n+1}$ and $\mathbb{S}^{n+1}$}

Let $\mathbb{N}^{n+1}(K)$ be a space form of constant sectional curvature $K$. There is corresponding notion of qumermassintegrals $\mathcal{A}_{k}(\Omega)$ for convex domain $\Omega$ in $\mathbb{N}^{n+1}(K)$ (e.g., [27]). If the 
boundary $\partial \Omega$ is $C^{2}$, it holds Cauchy-Cronfton formula,

$$
\begin{aligned}
& \mathcal{A}_{-1}(\Omega)=\mathcal{V}(\Omega), \quad \mathcal{A}_{0}(\Omega)=\int_{\partial \Omega} d \mu_{g}, \quad \mathcal{A}_{1}(\Omega)=\int_{\partial \Omega} \sigma_{1}(\kappa) d \mu_{g}+n K \mathcal{V}(\Omega), \\
& \mathcal{A}_{k}(\Omega)=\int_{\partial \Omega} \sigma_{k}(\kappa) d \mu_{g}+K \frac{n-k+1}{k-1} \mathcal{A}_{k-2}(\Omega), \quad k=2, \cdots, n-1 .
\end{aligned}
$$

More importantly, these geometric quantities enjoy the same variational property as in $\mathbb{R}^{n+1}$ (e.g., [2]),

$$
\delta_{\eta} \mathcal{A}_{k}(\Omega)=(k+1) \int_{\partial \Omega} \sigma_{k+1}(\kappa) f d \mu,
$$

for any variational vector field $\eta=f v$. Recall the Minkowski Identity (2.5) discussed in Section 2,

$$
\int_{M}\left[\sigma_{k+1}(\kappa) u-\frac{n-k}{k+1} \phi^{\prime}(\rho) \sigma_{k}(\kappa)\right] d \mu=0 .
$$

For general warped product spaces, Minkowski identity (4.3) involves a term related to Ricci tensor of the ambient space, due to lack of Codazzi properties (2.11).

Suppose we want to compare $\mathcal{A}_{l}$ and $\mathcal{A}_{k}$ for given $k<l$, suppose geodesic balls $B_{r}, 0, r>$ 0 in $\mathbb{N}^{n+1}(K)$ are the optimal solutions of this isoperimetric problem. Let $\xi_{l, k}(s)$ be the unique positive function such that

$$
\sim_{l, k}\left(A_{k}\left(B_{r}\right)\right)=\mathcal{A}_{l}\left(B_{r}\right), \quad \forall r>0 .
$$

Then we want to establish

$$
\sim_{l, k}\left(A_{k}(\Omega)\right) \leq \mathcal{A}_{l}(\Omega),
$$

with equality holds if and only if $\Omega$ is a geodesic ball.

As in the case of $\mathbb{R}^{n+1}$, the divergence identity (4.2) leads us to the following flow $\mathbb{N}^{n+1}(K)$ in [4],

$$
\partial_{t} X=\left(\phi^{\prime} \frac{\sigma_{k}}{\sigma_{k+1}}-\frac{u}{c_{n, k}}\right) v=\frac{\operatorname{div} W}{\sigma_{k+1}} v
$$

where the vector field $W=\sigma_{k}^{i j} \nabla_{j} \Phi$ as in (1.5). The quermassintegrals (4.1) enjoy the following monotonicity property,

$$
\frac{d}{d t} \mathcal{A}_{l}= \begin{cases}\geq 0, & \text { if } l<k \\ =0, & \text { if } l=k \\ \leq 0, & \text { if } l>k\end{cases}
$$

for all $0 \leq l \leq n-1$.

In order to prove (4.5) for $l=k+1$, one needs to establish the long time existence and convergence of the flow in the corresponding space form. 
The hypersurface flow equation (4.6) is equivalent to the initial value problem of a positive radial function $\rho$ on sphere $\mathbb{S}^{n}$,

$$
\left\{\begin{array}{l}
\partial_{t} \rho=\left(\frac{\phi^{\prime}}{F}-\frac{u}{c_{n}}\right) \omega, \quad \text { for } \quad(z, t) \in \mathbb{S}^{n} \times[0, \infty), \\
\rho(\cdot, 0)=\rho_{0},
\end{array}\right.
$$

where $\rho$ is the radial function and $F=\frac{\sigma_{k+1}}{\sigma_{k}}$ for simplicity. Let function $\gamma$ be defined as

$$
\frac{d \gamma}{d \rho}=\frac{1}{\phi}
$$

The corresponding evolution equation for $\gamma$ can be written as

$$
\partial_{t} \gamma=\frac{\phi^{\prime}}{u F}-\frac{1}{c_{n, k}} .
$$

By examining the evolution equation of $\rho$ or $\gamma$, the standard maximum principle yields $\mathrm{C}^{0}$ estimate

$$
\min _{x \in M} \rho(x, 0) \leq \rho(x, t) \leq \max _{x \in M} \rho(x, 0) .
$$

Straightforward computation yields

$$
\begin{aligned}
\mathcal{L}\left(\frac{h_{j}^{i}}{u}\right)=\frac{\phi^{\prime}}{u F^{2}} F^{k l ; p q} \nabla^{i} h_{p q} \nabla_{j} h_{k l}+\frac{1}{c_{n}} \nabla \frac{h_{j}^{i}}{u} \nabla \Phi+\frac{2}{u} \cdot \frac{\phi^{\prime}}{F^{2}} F^{k l} \nabla_{k} \frac{h_{j}^{i}}{u} \nabla_{l} u \\
+\frac{1}{2 u F \phi^{\prime}} \nabla^{i} \phi^{\prime} \nabla_{j} \phi^{\prime}-\frac{2 \phi^{\prime}}{u F}\left(\frac{\nabla^{i} F}{F}-\frac{\nabla^{i} \phi^{\prime}}{2 \phi^{\prime}}\right)\left(\frac{\nabla_{j} F}{F}-\frac{\nabla_{j} \phi^{\prime}}{2 \phi^{\prime}}\right) \\
-\frac{2 \phi^{\prime}}{u F}\left(h^{2}\right)_{j}^{i}+\left[-K \frac{\phi^{\prime}}{F^{2}} F^{k k}-K \frac{u}{F}+\frac{2 \phi^{\prime}}{c_{n}}+\frac{1}{u F} \nabla \Phi \nabla \phi^{\prime}\right] \frac{h_{j}^{i}}{u}+K\left(\frac{\phi^{\prime}}{u F}+\frac{1}{c_{n}}\right) \delta_{j}^{i},
\end{aligned}
$$

where $\mathcal{L}:=\partial_{t}-\frac{\phi^{\prime}}{F^{2}} F^{k l} \nabla_{k} \nabla_{l}$ is the linearized parabolic operator.

From now on, we will discuss (4.6) separately for $\mathbb{H}^{n+1}$ and for $\mathbb{S}^{n+1}$, due to different PDE issues involved for these cases.

\subsection{The Case $\mathbb{N}^{n+1}=\mathbb{H}^{n+1}$}

The main problem here is preservation of starshapedness of flow (4.6), equivalently, the gradient estimate for the corresponding flow (4.7) or flow (4.8). Let

$$
\mathcal{L}:=\partial_{t}-\frac{\cosh (\rho)}{\omega^{2} F^{2}} F^{i j}\left(\sinh ^{2}(\rho) \omega^{2} \delta_{i k}-\gamma_{i} \gamma_{k}\right) \partial_{k j}
$$

be the linearized operator of flow (4.8), where $\omega=\sqrt{1+|\nabla \gamma|^{2}}$. 
At maximum point of $\partial_{t} \gamma$

$$
\mathcal{L}\left(\gamma_{t}\right)=-\frac{\sinh (\rho) \sum F^{i i}}{\omega^{3} F} \gamma_{t}\left(\gamma_{t}+\frac{1}{c_{n, k}}-\frac{\omega^{2}}{\sum F^{i i}}\right) .
$$

(4.12) provides a lower bound of $F \geq C_{0}>0$ for all $t>0$ provided that $|\nabla \gamma|$ is uniformly bounded. Bound of $|\nabla \gamma|$ is equivalent to uniform lower bound of $u$. (4.10) and the maximum principle yield the curvature estimates which is equivalent to $C^{2}$ estimates.

$$
\max _{(x, t) \in \mathbb{S}^{n} \times[0, T)}\left|\nabla^{2} \rho\right|(x, t) \leq C,
$$

where $C$ is a constant depending on the uniform lower and upper bound of $\rho$ and upper bound of $|\nabla \rho|$.

The main issue is the gradient estimate which is still open for equation (4.8). We compute evolution equation for $|\nabla \gamma|^{2} / f(\gamma)$ where $f(\gamma)>0$ is any smooth positive function. Let $\mathcal{L}$ be the linearized operator as in (4.11). Then at the maximum point of $|\nabla \gamma|^{2} / f$, with the assumption that $\nabla \gamma=\gamma_{1}$,

$$
\begin{aligned}
\mathcal{L}\left(\ln \frac{|\nabla \gamma|^{2}}{f}\right)= & \frac{f^{\prime}}{c_{n} f \phi^{2} \omega^{6} F^{2}}\left(\phi \omega^{3} F-c_{n} \omega^{2} \cosh \rho\right)^{2}-\frac{2 \cosh \rho \sum_{j \geq 2} F^{j j}}{\phi^{2} F^{2}|\nabla \gamma|^{2}}\left(\gamma_{j j}+\frac{\sinh ^{2} \rho|\nabla \gamma|^{2}}{2 \cosh \rho}\right)^{2} \\
& +\frac{\cosh \rho}{2 \phi^{2} F^{2}} \sum_{j \geq 2} F^{j j}\left(\frac{\sinh ^{4} \rho}{\cosh ^{2} \rho}|\nabla \gamma|^{2}-4\right)+\frac{f^{\prime} \cosh ^{2} \rho}{f \omega^{2} \phi^{2} F^{2}}\left(\sum_{i=1}^{n} F^{i i}-c_{n}\right)+\frac{f^{\prime}}{f} \frac{1}{\omega^{2} \phi^{2} F^{2}}|\nabla \gamma|^{2} F^{11} \\
& +\frac{f^{\prime}}{f} \frac{\cosh ^{2} \rho}{\omega^{2} \phi^{2} F^{2}}|\nabla \gamma|^{2} \sum_{j \geq 2} F^{j j}-|\nabla \gamma|^{2} \frac{\cosh \rho}{\omega^{4} \phi^{2} F^{2}} F^{11}\left(\frac{1}{2}\left(\frac{f^{\prime}}{f}\right)^{2}\left(3|\nabla \gamma|^{2}+1\right)-\frac{f^{\prime \prime}}{f} \omega^{2}\right)
\end{aligned}
$$

where $\phi=\sinh \rho$.

Though we are not able to get gradient estimate in full, with the help of (4.14), the following can be proved under certain initial condition by letting $f(\gamma)=\cosh ^{2} \rho / \sinh ^{4} \rho$.

Proposition 4.1. Suppose $\gamma(\cdot, t)$ solves the corresponding initial value problem (4.7) on $\mathbb{S}^{n}$ for $t \in[0, T)$. Assume the initial hypersurface satisfies the following condition,

$$
\text { Condition }(C): \max _{x \in S^{n}}|\nabla \ln (\cosh \rho)|^{2}(x, 0) \leq 12+3 \min _{t=0} \sinh ^{2} \rho,
$$

Then this upper bound is uniform for all $t>0$, i.e.,

$$
\max _{(x, t) \in \mathbb{S}^{n} \times[0, T)}|\nabla \ln (\cosh \rho)|^{2}(x, t) \leq 12+3 \min _{t=0} \sinh ^{2} \rho .
$$

By standard parabolic PDE theory, after established $C^{0}, C^{1}$, and $C^{2}$ estimates, we obtained all the a priori estimates under Condition (4.15) and in turn the long time convergence of the flow. Also, in the special case that $k=n-1$, namely along the following flow

$$
\partial_{t} X=\left(\phi^{\prime} \frac{\sigma_{n-1}}{\sigma_{n}}-\frac{u}{c_{n, n-1}}\right) v
$$


since convexity implies gradient estimates. With that, the speed function is bounded from below. This yields that the convexity of hypersurfaces are preserved along flow (4.6). Hence, one has all the a priori estimates and convergence.

Theorem 4.1. Let $M_{0}$ be a radial graph of function $\rho_{0}$ over $\mathbb{S}^{n}$ in $\mathbb{H}^{n+1}$. Suppose either $k=n-1$, or Condition (4.15) is satisfied. Then flow (4.6) exists all time and convergent exponentially to a geodesic sphere. To be precise, solution $\gamma(z, t)$ of $(4.8)$ exists in interval $[0, \infty)$, and there exist a uniform constant $\alpha>0$ which depends only on the initial graph, such that for any $(z, t) \in$ $\mathrm{S}^{n} \times[0, \infty]$,

$$
e^{\alpha t}|\nabla \gamma|^{2}(z, t) \leq \max _{z \in \mathrm{S}^{n}}|\nabla \gamma|^{2}(z, 0),
$$

where the covariant derivatives are with respect to the spherical metric on $\mathbb{S}^{n}$.

As a consequence, sharp isoperimetric inequality comparing $\mathcal{A}_{n-1}$ with all other $\mathcal{A}_{k}$ for convex domains in $\mathbb{H}^{n+1}$ can be proved. In two other special cases, we can manage to get around and obtain sharp quermassintegral inequalities. More specifically, we can compare the first and second quermassintegrals, $\mathcal{A}_{2}$ and $\mathcal{A}_{1}$, with $\mathcal{A}_{0}$ respectively.

In the case of $h$-convexity, full range of quermassintegral inequalities were obtained in $[10,31]$ using contracting type of flows. Very recently, the results in [31] for $h$-convex domains in $\mathbb{H}^{n+1}$ were reproved using flow (4.6) directly in [21] by establishing that $h$ convexity is preserved along flow (4.6). The sharp relation between $\mathcal{A}_{2}$ and $\mathcal{A}_{0}$ was previously proved in [24] by a different method.

Recall the inverse mean curvature flow

$$
\partial_{t} X=\frac{1}{H} v
$$

and inverse curvature flow

$$
\partial_{t} X=\frac{\sigma_{1}}{\sigma_{2}} v
$$

studied in [12] (see also [8]).

Lemma 4.1. Let $M(t)$ be a smooth family of hypersurfaces.

1. If $M(t)$ solves the inverse mean curvature flow equation (4.19) with initial condition $M(0)=$ $M$, then

$$
e^{-\frac{n-1}{n} t}\left(\mathcal{A}_{1}(t)-\xi_{1,0}\left(\mathcal{A}_{0}(t)\right)\right) \leq \mathcal{A}_{1}(0)-\xi_{1,0}\left(\mathcal{A}_{0}(0)\right) \text {. }
$$

2. If $M(t)$ solves the inverse curvature flow equation (4.20) with initial condition $M(0)=M$, then

$$
e^{-\frac{2(n-2)}{n-1} t}\left(\mathcal{A}_{2}(t)-\xi_{2,0}\left(\mathcal{A}_{0}(t)\right)\right) \leq \mathcal{A}_{2}(0)-\xi_{2,0}\left(\mathcal{A}_{0}(0)\right) .
$$


We provide two proofs for the sharp geometric inequality between $\mathcal{A}_{1}$ and $\mathcal{A}_{0}$ for a star-shaped domain $\Omega \subset \mathbb{H}^{n+1}$ with smooth boundary. Notice that if the boundary hypersurface satisfies the gradient bounds in Theorem 4.1, then the sharp inequality follows immediately from the long time existence and exponential convergence of flow (4.6) with $k=1$.

As a conclusion, we have the following sharp geometric inequalities.

Theorem 4.2. (44]) Suppose $\Omega$ is a bounded domain in $\mathbb{H}^{n+1}$ with smooth boundary. The following three results hold:

1. When $l=1$ or $l=2$, if $\Omega$ is starshaped and $l$-convex, then

$$
\sim_{l, 0}\left(A_{0}(\Omega)\right) \leq \mathcal{A}_{l}(\Omega) .
$$

2. For all $l=-1,0, \cdots, n-2$, if $\Omega$ is convex,

$$
\sim_{n-1, l}\left(A_{l}(\Omega)\right) \leq \mathcal{A}_{n-1}(\Omega) .
$$

3. In general, if $\partial \Omega$ satisfies Condition (4.15), then for all $k<l, l, k=0, \cdots, n-1$,

$$
\sim_{l, k}\left(A_{k}(\Omega)\right) \leq \mathcal{A}_{l}(\Omega) .
$$

Equality holds if and only if $\Omega$ is a geodesic ball.

Case (1) in the theorem for $l=2$ was proved in [24] using different flow. We believe Condition (4.15) is redundant in above Theorem. We also refer [5] for the Minkowski inequality in the anti-de Sitter-Schwarzschild space.

Proof. Cases (2) and (3) follow from the longtime existence and convergence of flow (4.6) under Condition (4.15) or for $k=n-1$. We provide two proofs for $l=1$ of Case (1).

Proof 1. We wixll combine flow (4.6) and inverse mean curvature flow to complete the proof. Let $M(t)$ be a solution to the inverse mean curvature flow (4.19) with initial condition $M(0)=\partial \Omega$. By Gerhardt's estimate for the radial function $\rho(t)$, there exists a large enough $T>>0$, such that

$$
|\nabla \log \cosh \rho(T)| \leq \min _{x \in M(T)} \sinh ^{2} \rho(T) .
$$

By Proposition 4.1, we have

$$
\mathcal{A}_{1}(M)-\xi_{1,0}\left(\mathcal{A}_{0}(M)\right) \geq e^{-\frac{n-1}{n} T}\left(\mathcal{A}_{1}(M(T))-\xi_{1,0}\left(\mathcal{A}_{0}(M(T))\right)\right) .
$$

Let $\tilde{M}(t)$ be a solution to the modified inverse mean curvature flow

$$
\partial X=\left(\frac{\cosh \rho}{H}-\frac{u}{n}\right) v
$$


with initial condition $\tilde{M}(0)=M(T)$. Notice that (4.25) is a special case of flow (4.6) when $k=1$. Notice that $\tilde{M}(0)=M(T)$ which satisfies the gradient bounds in Theorem 4.1, then the following sharp inequality

$$
\mathcal{A}_{1}(\tilde{M}(0))-\xi_{1,0}\left(\mathcal{A}_{0}(\tilde{M}(0))\right) \geq 0
$$

follows from the long time existence, exponential convergence of flow (4.6) with $k=1$, and the monotonicity formulas of $\mathcal{A}_{k}$ along the flow. Since $\tilde{M}(0)=M(T)$, combining (4.24) and (4.26), we finish the proof.

Proof 2. The following argument is due to Simon Brendle, using inverse mean curvature flow directly to prove the inequality.

Let now $\hat{\Sigma}_{t}$ be a family of geodesic spheres evolving under inverse mean curvature flow with $\left|\hat{\Sigma}_{t}\right|=\left|\Sigma_{t}\right|$. Moreover, since $\frac{d}{d t}\left|\Sigma_{t}\right|=\left|\Sigma_{t}\right|$, we have

$$
\left|\hat{\Sigma}_{t}\right|=e^{t}\left|\hat{\Sigma}_{0}\right|=e^{t}\left|\Sigma_{0}\right|
$$

Recall

$$
\mathcal{A}_{1}\left(\Sigma_{t}\right)=\int_{\Sigma_{t}} H d \mu-n V(t) .
$$

When $t$ is large enough, we have along the inverse MCF,

$$
H=n+O\left(t e^{-\frac{2}{n} t}\right) .
$$

Thus

$$
\begin{aligned}
\mathcal{A}_{1}\left(\Sigma_{t}\right) & =n\left|\Sigma_{t}\right|-n V(t)+O\left(t e^{-\frac{2}{n} t}\right)\left|\Sigma_{t}\right| \\
& \geq n\left|\Sigma_{t}\right|-n \xi_{-1}\left(\left|\Sigma_{t}\right|\right)+O\left(t e^{-\frac{2}{n} t}\right)\left|\Sigma_{t}\right| \\
& =n\left|\hat{\Sigma}_{t}\right|-n \xi_{-1}\left(\left|\hat{\Sigma}_{t}\right|\right)+O\left(t e^{-\frac{2}{n} t}\right)\left|\hat{\Sigma}_{t}\right| \\
& =n\left|\hat{\Sigma}_{t}\right|-n V\left(\hat{\Sigma}_{t}\right)+O\left(t e^{-\frac{2}{n} t}\right)\left|\hat{\Sigma}_{t}\right| \\
& =\mathcal{A}_{1}\left(\hat{\Sigma}_{t}\right)+O\left(t e^{-\frac{2}{n} t}\right)\left|\hat{\Sigma}_{t}\right|,
\end{aligned}
$$

where the inequality follows from isoperimetric inequality in hyperbolic space, the second identity follows from the fact that $\left|\hat{\Sigma}_{t}\right|=\left|\Sigma_{t}\right|$, the third identity follows from the equality case of sharp isoperimetric inequality in hyperbolic space, and the last identity follows from the definition.

Since $\mathcal{A}_{0}\left(\Sigma_{t}\right)=\left|\Sigma_{t}\right|$, we have $\xi_{1}\left(\mathcal{A}_{0}(t)\right)=\xi_{1}\left(\left|\Sigma_{t}\right|\right)=\xi_{1}\left(\left|\hat{\Sigma}_{t}\right|\right)$. Thus,

$$
\mathcal{A}_{1}\left(\Sigma_{t}\right)-\xi_{1,0}\left(\mathcal{A}_{0}\left(\Sigma_{t}\right)\right) \geq \mathcal{A}_{1}\left(\hat{\Sigma}_{t}\right)+O\left(t e^{-\frac{2}{n} t}\right)\left|\hat{\Sigma}_{t}\right|-\xi_{1,0}\left(\left|\hat{\Sigma}_{t}\right|\right)=O\left(t e^{-\frac{2}{n} t}\right)\left|\hat{\Sigma}_{t}\right|,
$$

where we used $\mathcal{A}_{1}\left(\hat{\Sigma}_{t}\right)-\xi_{1,0}\left(\left|\hat{\Sigma}_{t}\right|\right)=0$ on geodesic spheres. 
We have for large enough $t$,

$$
\begin{aligned}
& e^{-\frac{n-1}{n} t}\left(\mathcal{A}_{1}(t)-\xi_{1,0}\left(\mathcal{A}_{0}(t)\right)\right) \\
\geq & e^{-\frac{n-1}{n} t} O\left(t e^{-\frac{2}{n} t}\right)\left|\hat{\Sigma}_{t}\right|=e^{-\frac{n-1}{n} t} O\left(t e^{-\frac{2}{n} t}\right) e^{t}\left|\Sigma_{0}\right|=O\left(t e^{-\frac{1}{n} t}\right),
\end{aligned}
$$

where in the second identity, we have used the evolution of area functional under inverse $\mathrm{MCF}$, i.e. $A^{\prime}(t)=A(t)$.

By (4.21), this finishes the proof of

$$
0 \leq \mathcal{A}_{1}(0)-\xi_{1,0}\left(\mathcal{A}_{0}(0)\right)
$$

using the monotonicity.

The proof of $l=2$ in Case (1) is similar to the Proof 1.

\subsection{The case $\mathbb{N}^{n+1}=\mathbb{S}_{+}^{n+1}$}

We already have $C^{0}$ estimate (4.9). With $\phi(\rho)=\sin \rho, \phi^{\prime}(\rho)=\cos \rho$, the support function $u=<X, v>$ evolves as following respectively,

$$
\mathcal{L}(u)=\frac{|\nabla \rho|^{2} \sin ^{2} \rho}{F}-\frac{1}{c_{n, k}} \nabla \cos \rho \nabla u+\left(\frac{1}{F^{2}} F^{i j}\left(h^{2}\right)_{i j}-\frac{1}{c_{n, k}}\right) u \cos \rho,
$$

where $\mathcal{L}=\partial_{t}-\frac{\phi^{\prime}}{F^{2}} F^{i j} \partial_{i j}$. The lower bound of $u \geq c_{0}>0$ follows immediately in this case. Then it follows (4.10) and concavity of $F$, one obtains curvature bound of flow (4.6) in $\mathrm{S}_{+}^{N+1}$ : there is $C>0$ depending only on the initial data such that,

$$
\max _{M(t)} h_{j}^{i} \leq C, \quad \forall i, j, \text { hence }\|\rho\|_{C^{2}\left(\mathbb{S}^{n}\right)} \leq C .
$$

For the longtime existence and exponential convergence of flow (4.6) in $S_{+}^{n+1}$, we need the key estimate of the lower bound of $F$

$$
F \geq C_{0}>0
$$

Unfortunately, estimate (4.29) is still open, except for the special case $k=n-1$.

Proposition 4.2. Suppose $k=n-1$ and $\Omega_{0}$ is strictly convex, and suppose $\partial \Omega(t)$ is the solution of flow (4.6) in $S_{+}^{n+1}$, then

$$
\min _{\partial \Omega(t)} u F \geq \min _{\partial \Omega_{0}} u F
$$

That is, $u F$ is bounded from below by a positive constant depending only on initial data. As a consequence, $F$ is bounded from below uniformly and convexity is preserved. 
Proof. One computes that

$$
\mathcal{L}(u F)=-\frac{2}{F} F^{i j} \nabla^{i} \phi^{\prime} \nabla_{j} u+|\nabla \Phi|^{2}+u^{2}\left(\frac{\sum F^{i i}}{c_{n, k}}-1\right)+O(|\nabla(u F)|) .
$$

Note $\Phi=-\phi^{\prime}=-\cos \rho$, and $u_{i}=h_{i}^{j} \Phi_{j}=-h_{i}^{j} \phi_{j}^{\prime}$. When $t>0$ small, $h_{i}^{j}>0$, by (4.31), at minimal point of $u F, \mathcal{L}(u F) \geq 0$. Since the lower bound of $F$ is independent of $t$, preservation of convexity follows directly as $n=k+1$.

Theorem 4.3 ([4]). Suppose $\partial \Omega_{0}$ is strictly smooth convex domain in $\mathrm{S}_{+}^{n+1}$, then the evolution equation (4.6) with $k=n-1$ has a smooth solution for $t \in[0, \infty)$. Moreover, the hypersurfaces converge exponentially to a geodesic sphere as $t \rightarrow \infty$ in the $C^{\infty}$ topology. As a consequence,

$$
\sim_{n-1, k}\left(A_{k}\left(\Omega_{0}\right)\right) \leq \mathcal{A}_{n-1}\left(\Omega_{0}\right), \quad \forall k \leq n-1,
$$

$"="$ holds if and only if $\Omega_{0}$ is a geodesic ball.

We also consider the following fully nonlinear version of (2.2) in $S_{+}^{n+1}$ in [6],

$$
\partial_{t} X=\left(c_{n, k} \phi^{\prime}-\frac{\sigma_{k+1}}{\sigma_{k}} u\right) v
$$

Similar to all other flows in this article, monotonicity formulas hold for the quermassintegrals in $\mathrm{S}_{+}^{n+1}$ as long as the flow exists. Flow (4.33) preserves convexity, and one has $C^{1}$ estimates for solutions, and upper and lower bounds of $F=\sigma_{k+1} / \sigma_{k}$ along flow (4.33). Except in the case of $k=0$ (i.e., flow (2.2)) where $C^{2}$ estimates follows directly from the theory of quasi-linear PDE, $C^{2}$ estimates for solutions of flow (4.33) is still an open question.

Flow (4.33) is the same as flow (3.5) in $\mathbb{R}^{n+1}$ where $\phi^{\prime}=1$. In Section 3, we convert (3.5) to a parabolic equation (3.6) on $\mathbb{S}^{n}$. It's relative easier to work on (3.6) for admissible solutions, even though we don't have curvature estimate for the original equation (3.5). One would like to search a similar transformation for flow (4.33) in $S_{+}^{n+1}$.

\subsection{Conclusion remarks}

Flow approach for geometric inequalities is not new, however the constraint hypersurface flows discussed here for isoperimetric problems are different from previous works. The guiding idea is to use variational properties of the concerned geometric functionals $\mathcal{F}$ and $\mathcal{G}$ along variational field $\eta=f v$,

$$
\delta_{\eta} \mathcal{F}=\int_{M} f P d \mu, \quad \delta_{\eta} \mathcal{G}=\int_{M} f Q d \mu,
$$

to design a flow of the form

$$
X_{t}=f v .
$$


Then $f$ must be of the form $f=\frac{\operatorname{div} W}{P}$ for some vector field $W$ if $\mathcal{F}$ is preserved. The crucial step is to make proper choice of vector field $W$ so that the functional $\mathcal{G}$ is monotone along the flow, i.e.,

$$
\int_{M} \frac{Q \operatorname{div} W}{P} d \mu \geq 0 \quad(\text { or } \quad \leq 0) .
$$

For quermassintegrals, $W$ in (1.5) is a natural choice where the conformal vector field $V=\phi \frac{\partial}{\partial \rho}$ plays key role. This choice leads to mean curvature type or inverse mean curvature type flows. The PDE problems arising from these flows are interesting and challenging. Resolution of these PDE problems have significant geometric implications. General principles outlined here can be extended to treat other geometric problems in other settings (e.g. [29,32] and [23, 28]).

\section{Acknowledgments}

Part of this article (proof in Section 3) was completed while the xfirst author was visiting RIMS, Kyoto University. He would like to thank Professor Kaoru Ono for hosting him and thank RIMS for the warm hospitality.

\section{References}

[1] B. Andrews, Y. Hu and H. Li, Harmonic mean curvature flow and geometric inequalities, arXiv:1903.05903.

[2] J. Barbosa and A. G. Colares, Stability of hypersurfaces with constant r-mean curvature, Ann. Global Anal. Geom., 15(3) (1997), 277-297.

[3] S. Brendle, Constant mean curvature surfaces in warped product manifolds, Publ. Math. Inst. Hautes Études Sci., 117 (2013), 247-269.

[4] S. Brendle, P. Guan and J. Li, An inverse curvature type hypersurface flow in space forms, Preprint.

[5] S. Brendle, P. K. Hung and M. T. Wang, A Minkowski inequality for hypersurfaces in the anti-de Sitter-Schwarzschild manifold, Commun. Pure Appl. Math., 69(1) (2016), 124-144.

[6] C. Chen, P. Guan and J. Li et al., A curvature hypersurface flow in $\mathrm{S}_{+}^{n+1}$, work in progress.

[7] L. L. De Lima and F. Girao, An Alexandrov-Fenchel-type inequality in hyperbolic space with an application to a Penrose inequality, Ann. Henri Poincarè, 17(4) (2016), 979-1002.

[8] Q. Ding, The inverse mean curvature flow in rotationally symmetric spaces, China Ann. Math. Ser. B, 32(1)(2011), 27-44.

[9] M. Gage and R. Hamilton, The heat equation shrinking convex plane curves, J. Differential Geom., 23(1) (1986), 69-96.

[10] Y. Ge, G. Wang and J. Wu, Hyperbolic Alexandrov-Fenchel quermassintegral inequalities II, J. Differential Geom., 98(2) (2014), 237-260.

[11] C. Gerhardt, Flow of nonconvex hypersurfaces into spheres, J. Differential Geom., 32 (1990), 299-314.

[12] C. Gerhardt, Inverse curvature flows in hyperbolic space, J. Differential Geom., 89(3) (2011), 487-527. 
[13] P. Guan, Curvature measures, isoperimetric inequalities and fully nonlinear PDEs, Fully Nonlinear PDEs in Real and Complex Geometry and Optics, Cetraro, Italy, 2012, Editors: Cristian E. Gutiarrez, Ermanno Lanconelli, Springer, 2014, 47-94.

[14] P. Guan and J. Li, The quermassintegral inequalities for $k$-convex starshaped domains, Adv. Math., 221(5) (2009), 1725-1732.

[15] P. Guan and J. Li, A mean curvature type flow in space forms, Int. Math. Res. Not. IMRN, 13 (2015), 4716-4740.

[16] P. Guan and J. Li, A fully-nonlinear flow and quermassintegral inequalities, Scientia Sinica Math., 48(1) (2018).

[17] P. Guan, J. Li and M. T. Wang, A volume preserving flow and the isoperimetric problem in warped product spaces, Trans. Am. Math. Soc., 372 (2019), 2777-2798.

[18] P. Guan and G. Wang, A fully nonlinear conformal flow on locally conformally flat manifolds, J. Reine Angew. Math., 557 (2003), 219-238.

[19] P. Guan and G. Wang, Geometric inequalities on locally conformally flat manifolds, Duke Math. J., 124 (2004), 177-212.

[20] E. Heintze and H. Karcher, A general comparison theorem with applications to volume estimates for submanifolds, Ann. Sci. École Norm Sup., 11 (1978), 451-470.

[21] Y. Hu, H. Li and Y. Wei, Locally constraint inverse curvature flows in hyperbolic space, Preprint.

[22] G. Huisken, Flow by mean curvature of convex surfaces into spheres, J. Differential Geom., 20 (1984), 237-266.

[23] B. Lambert and J. Scheuer, Isoperimetric problems for spacelike domains in generalized Robertson-Walker spaces, https://arXiv.org/abs/1910.06696.

[24] H. Li, Y. Wei and C. Xiong, A geometric inequality on hypersurface in hyperbolic space, Adv. Math., 253 (2014), 152-162.

[25] J. Li and C. Xia, An integral formula and its applications on sub-static manifolds, arXiv:1603.02201, 2016.

[26] A. Ros, Compact hypersurfaces with constant higher order mean curvatures, Revista Mathmática Iberoamericana, 3 (1987), 447-453.

[27] L. A. SantalÓ, Integral Geometry and Geometric Probability, Second editio, with a foreword by Mark Kac, Cambridge Mathematical Library, Cambridge University Press, Cambridge, 2004.

[28] J. Scheuer, The Minkowski inequality in de Sitter space, arXiv:1909.06837.

[29] J. Scheuer, G. Wang and C. Xia, Alexandrov-Fenchel inequalities for convex hypersurfaces with free boundary in a ball, arXiv:1811.05776.

[30] F. Schulze, Nonlinear evolution by mean curvature and isoperimetric inequalities, J. Differential Geom., 79(2) (2008), 197-241.

[31] G. Wang and C. Xia, Isoperimetric type problems and Alexandrov-Fenchel type inequalities in the hyperbolic space, Adv. Math., 259 (2014), 532-556.

[32] G. Wang, and C. Xia, Guan-Li type mean curvature flow for free boundary hypersurfaces in a ball, https://arXiv.org/abs/1910.07253. 